\title{
The cost-benefit of genomic testing of heifers and using sexed semen in pasture-based dairy herds
}

\author{
J. E. Newton, ${ }^{* 1}$ B. J. Hayes, ${ }^{*} \dagger$ and J. E. Pryce ${ }^{*} \ddagger$ \\ *Agriculture Victoria, AgriBio, Centre for AgriBioscience, Bundoora, VIC 3083, Australia \\ †Queensland Alliance for Agriculture and Food Innovation, University of Queensland, Brisbane, St. Lucia, QLD 4072, Australia \\ $\ddagger$ School of Applied Systems Biology, La Trobe University, Bundoora, VIC 3083, Australia
}

\begin{abstract}
Recent improvements in dairy cow fertility and female reproductive technologies offer an opportunity to apply greater selection pressure to females. This means there may be greater incentive to obtain genomic breeding values for females. We modeled the impact of changes to key parameters on the net benefit from genomic testing of heifer calves with and without usage of sexed semen. This paper builds on earlier cost-benefit studies but uses parameters relevant to pasture-based systems. A deterministic model was used to evaluate the effect on net benefit due to changes in (1) reproduction rate, (2) genomic test costs, (3) availability of parent-derived breeding values $\left(\mathrm{EBV}_{\mathrm{PA}}\right)$, and (4) replacement rate. When the use of sexed semen was included, we also considered (1) the proportion of heifers and cows mated to sexed semen, (2) decreases in conception rate in inseminations with sexed semen, and (3) the marginal return for surplus heifers. Scenarios with lower replacement rates and no availability of $\mathrm{EBV}_{\mathrm{PA}}$ had the largest net benefits. Under current Australian parameters, the net benefit of genomic testing realized over the lifetime of genotyped heifers is expected to range from $\mathrm{A} \$ 204$ to $\mathrm{A} \$ 1,124$ per 100 cows for a herd with median reproductive performance. The cost of a genomic test, a perceived barrier to many farmers, had only a small effect on net benefit. Genomic testing alone was always more profitable than using sexed semen and genomic testing together if the only benefit considered was increased genetic gain in heifer replacements. When other benefits (i.e., the higher sale price of a surplus heifer compared with a male calf) were considered, there were combinations of parameters where net benefit from using sexed semen and genomic testing was higher than the equivalent scenario with genomic testing only. Using sexed semen alongside genomic testing is most likely to be
\end{abstract}

Received July 11, 2017.

Accepted March 5, 2018.

${ }^{1}$ Corresponding author: jo.newton@ecodev.vic.gov.au profitable when (1) used in heifers, (2) the marginal return for selling surplus heifers (sale price minus rearing costs) is greater than $\mathrm{A} \$ 400$, and (3) conception rates of no more than 10 percentage points lower than those achieved using conventional semen can be realized. Net benefit was highly dependent on the marginal return. Demonstrating that the initial investment in genomic testing can be recouped within the lifetime of the heifers tested may assist in the development of extension messages to explain the value of genomic testing females at the herd level.

Key words: genomic testing, rate of genetic gain, sexed semen

\section{INTRODUCTION}

The commercialization of genomic testing has irrevocably changed dairy cattle breeding schemes. The high reliabilities being achieved for EBV derived using genomics $\left(\mathbf{E B V}_{\mathbf{G}}\right)$, coupled with reductions in the cost of genomic testing, have seen the number of animals evaluated using genomic data increase exponentially. For instance, at the beginning of 2009, shortly after genomic testing was first offered commercially in the United States, fewer than 20,000 animals had been tested. Today, over 211,000 Holstein bulls and over 1.2 million Holstein cows have been evaluated using genomics in the United States (https://www.uscdcb.com/ Genotype/cur_density.html; accessed April 2017).

Genomic testing of commercial dairy females benefits genetic evaluations of populations through regular contributions to reference populations (Mc Hugh et al., 2011; Pryce et al., 2012). Herd-level benefits include (1) more reliable decisions in selecting herd replacements, (2) fewer errors in parentage assignment, (3) the development of more targeted breeding objectives, and (4) earlier identification of candidates to artificially inseminate (AI) to high-value semen or for use in embryo transfer and in vitro fertilization programs.

Relatively few studies consider the cost-benefit of genomic testing of commercial dairy heifers at a herd 
level and many of these vary widely in their estimates of the benefits. For example, when considering the value of genomic testing over selection based on the average of parent EBV $\left(\mathbf{E B V}_{\mathbf{P A}}\right)$, Pryce and Hayes (2012) concluded that at a cost of $\mathrm{A} \$ 50$ per test (Australian dollars), genomic testing of heifers could not be justified. However, Weigel et al. (2012) concluded that, in most instances, the benefits exceeded the costs of genomic testing whether $\mathrm{EBV}_{\mathrm{PA}}$ were available or not. Both studies used the reliability of $\operatorname{EBV}_{\mathrm{PA}}\left(\boldsymbol{R}_{P A}\right)$ in their calculation of the benefit of selection using $\mathrm{EBV}_{\mathrm{G}}$ over $\mathrm{EBV}_{\mathrm{PA}}$. This is calculated from sire and dam reliabilities $\left(R_{s}\right.$ and $\left.R_{d}\right)$ as follows: $R_{P A}=0.25 \times\left(R_{s}+\right.$ $\left.R_{d}\right)$. Bijma (2012) showed that, in selected populations, doing this leads to an overestimation of the genetic progress possible, because the reliability of selection based on $\mathrm{EBV}_{\mathrm{PA}}$ is lower than the reliability of $\mathrm{EBV}_{\mathrm{PA}}$. When Calus et al. (2015) applied the correction suggested by Bijma (2012), they demonstrated that the benefits of selection using genomic-based strategies over pedigree-based selection strategies were likely to have been underestimated in earlier papers.

One of the challenges with genomic testing of female replacements is that there has been little room to apply selection pressure because most heifers are retained as replacements (Boichard et al., 2013). Recent indications are that selection for fertility is improving cow reproductive performance (Berry et al., 2014), meaning more replacements are being born. The use of sexed semen in AI programs also offers an opportunity to increase the number of heifer calves born and to intensify selection pressure on the female side (Boichard et al., 2013; Hjortø et al., 2015). Recent field trials show that sexed semen AI is starting to achieve conception rates more comparable to that of conventional AI (Healy et al., 2013; Butler et al., 2014; Izzo, 2015). Thus, using sexed semen in conjunction with genomic testing may be a profitable strategy.

Calus et al. (2015) found that the potential benefits from using $\mathrm{EBV}_{\mathrm{G}}$ instead of $\mathrm{EBV}_{\mathrm{PA}}$ were greater when sexed semen was used. However, they did not include the additional costs associated with using sexed semen (i.e., higher cost of straws and the cost of rearing additional heifer calves). In assessing the value of sexed semen, Fetrow et al. (2007) included many of these but did not put a value on genetic gain. They concluded that using sexed semen is unlikely to be cost effective unless genetic gain is considered. Although McCullock et al. (2013) included a genetic gain variable alongside other costs, the interaction between increased number of selection candidates (due to increased sexed semen usage) and genetic gain was not considered.

The net benefit from genomic testing is largely governed by the intensity with which selection can be ap- plied. At the herd level, this is influenced by parameters such as reproduction rate in heifers and cows and herd replacement rate. It is possible that the cost-benefit of genomic testing is different in pasture-based herds compared with confined systems reliant on TMR. For example, the number of calves born to AI sires versus non-AI herd bulls is a consideration in pasture-based herds. This is because for most pasture-based systems, it is general practice to have a defined period of mating to AI bulls followed by non-AI herd bulls.

The broad aim of this study was to evaluate the effect of changes to key herd parameters on the net benefit from genotyping heifers to inform selection decisions. We also evaluated whether the net benefit of genomic testing increased by concurrently adopting sexed semen with genomic selection. We build on earlier studies on the adoption of genomic testing either alone or in conjunction with sexed semen. In addition to using the formula of Bijma (2012) and accounting for discounting over time, we included parameters associated with reproduction rate as a simulation input. Using this instead of number of calves meant the effect of reduced conception rates associated with sexed semen could also be included.

\section{MATERIALS AND METHODS}

\section{Overview}

A deterministic simulation tool was developed in $\mathrm{R}$ version 3.2.3 (R Development Core Team, 2017) to estimate the net benefit from genotyping heifer selection candidates in commercial dairy herds. In total, 3 scenarios were considered, as described in Table 1. In brief, in scenario 1, only the adoption of genomic testing was considered. Scenarios 2 and 3 included the concurrent adoption of sexed semen to increase the number of heifer selection candidates alongside genomic testing. In scenario 2, only the cost of genomic testing and the additional cost of purchasing sexed semen were considered in the model. In scenario 3, the cost of genomic testing plus additional costs, losses, and benefits likely to be associated with the adoption of sexed semen were considered. All results are reported in Australian dollars (A\$); $\mathrm{A} \$ 1=\mathrm{US} \$ 0.80$ (http://www.xe.com/ currencyconverter/; accessed January 2018).

In the first scenario, we varied (1) reproduction rates, (2) genomic test costs, (3) availability of $\mathrm{EBV}_{\mathrm{PA}}$, and (4) replacement rates. Ranges and levels for each parameter varied are given in Table 2 . In the sexed semen scenarios (scenarios 2 and 3 ), the additional parameters that were varied were (1) the proportion of heifers or cows bred to sexed semen, and (2) the decrease in conception rate in inseminations with sexed semen. In 
Table 1. Overview of the 3 scenarios tested, the benefits and costs (and losses) considered, and the parameters that were varied for the 3 scenarios considered

\begin{tabular}{|c|c|c|c|c|}
\hline Scenario & Description & Costs (losses) & Benefits & Variables \\
\hline 2 & $\begin{array}{l}\text { Net benefit from genomic } \\
\text { testing heifer selection } \\
\text { candidates and using sexed } \\
\text { semen }\end{array}$ & $\begin{array}{l}\text { 1. Genomic test cost } \\
\text { 2. Sexed semen straw costs }\end{array}$ & $\begin{array}{l}\text { 1. Superiority of selected } \\
\text { heifers }\end{array}$ & $\begin{array}{l}\text { As for scenario } 1 \text { plus } \\
5 \text {. Proportion of heifers or cows } \\
\text { bred to sexed semen } \\
6 \text {. Decrease in conception rate } \\
\text { in inseminations with sexed } \\
\text { semen }\end{array}$ \\
\hline 3 & $\begin{array}{l}\text { Net benefit from genomic } \\
\text { testing heifer selection } \\
\text { candidates and using sexed } \\
\text { semen considering all } \\
\text { associated costs, benefits } \\
\text { and losses }\end{array}$ & $\begin{array}{l}\text { As for scenario } 2 \text { plus } \\
3 \text {. Lost income from male calf } \\
\text { sales } \\
\text { 4. Additional rebreeding costs } \\
\text { when conception rates lowered } \\
5 \text {. Lost milk income when } \\
\text { conception is delayed }\end{array}$ & $\begin{array}{l}\text { 1. Superiority of selected } \\
\text { heifers } \\
\text { 2. Sale of surplus heifers }\end{array}$ & $\begin{array}{l}\text { As for scenario } 2 \text { plus } \\
\text { 7. Marginal return for surplus } \\
\text { heifers }\end{array}$ \\
\hline
\end{tabular}

${ }^{1} \mathrm{EBV}_{\mathrm{PA}}=$ parent-derived $\mathrm{EBV}$.

scenario 3, we also varied (3) the marginal return for surplus heifer calves (MRH, i.e., the sale price minus rearing costs; Table 2). Further details are explained in the next section. Although the general assumptions and ranges of parameters tested in this model are representative of the conditions in pasture-based systems in Australia, it could easily be adapted with assumptions appropriate for other countries.

\section{Model Inputs and Assumptions}

For ease of interpretation, a Holstein herd with 100 cows was modeled. We assumed that genotyping took place 3 wk from birth, with all heifers reared to at least $3 \mathrm{mo}$ of age to allow time to receive genotyping results. Calf mortality rate from confirmation of dam pregnancy to weaning was assumed to be $10 \%$, whereas heifer mortality rate from weaning to first parturition was $5 \%$, and adult cow mortality was assumed to be $2.5 \%$. An overview of assumptions common to all 3 scenarios is given in Table 3 .

Herd Reproduction. Three different herd levels of reproductive performance were used: low, medium, and high (Table 4) and are consistent with the reproductive performance reported in a large study of Australian herds (Morton, 2011). The reproductive parameters used to define low, medium, and high reproductive performance correspond to the reproductive parameters of the bottom, median, and top quartiles of Australian dairy herds (Morton 2011). Reproductive performance parameters were separated by age group (heifers and cows) and mating type (AI or non-AI herd bull; Table

Table 2. Overview of the parameters varied in all 3 scenarios and the additional parameters considered in the sexed semen (SS) scenarios

\begin{tabular}{lrl}
\hline Parameter & $\begin{array}{c}\text { No. of } \\
\text { levels }\end{array}$ & Range \\
\hline Parameters for all scenarios & 3 & \multicolumn{2}{l}{ Low, medium, high } \\
Reproduction rate & Up to 25 & 16.9 to $40 \%$ \\
Replacement rate & 2 & A $\$ 40$ or A $\$ 50$ \\
Genomic test cost & 2 & No EBV available or prior availability of EBV ${ }_{\text {PA }}^{2}$ \\
Pedigree availability & 4 & $0-100 \%$ \\
Additional parameters for SS scenarios & 4 & $0-100 \%$ \\
Proportion of heifers undergoing AI with SS & 7 & $0-30$ percentage points \\
Proportion of cows undergoing AI with SS & 4 & A $\$ 0$ to A $\$ 600$ \\
Decrease in conception rate with SS vs. conventional semen & 4 \\
Marginal return for surplus heifers & & \\
\hline
\end{tabular}

${ }^{1}$ The range of replacement rates tested varied for each scenario; the minimum replacement rate was equivalent to the involuntary cull rate, the number of cows that exited the herd each year.

${ }^{2} \mathrm{EBV}_{\mathrm{PA}}=$ parent-derived EBV. 
Table 3. Underlying assumptions used in model across all 3 scenarios

\begin{tabular}{|c|c|}
\hline Parameter & Value \\
\hline \multicolumn{2}{|l|}{ Herd parameters } \\
\hline Herd size & 100 cows \\
\hline Age at first calving & $24 \mathrm{mo}$ \\
\hline Calf mortality rate (diagnosis of dam pregnancy to weaning) & 0.10 \\
\hline Heifer mortality rate (weaning to first calf) & 0.05 \\
\hline Cow mortality rate & 0.025 \\
\hline \multicolumn{2}{|l|}{ Sexed semen parameters ${ }^{1}$} \\
\hline Ratio of female to male calves in sexed semen & 0.9 \\
\hline Premium for sexed semen straw & $\mathrm{A} \$ 30$ \\
\hline Value of male calf & $\mathrm{A} \$ 54.30$ \\
\hline Value of lost milk income ${ }^{2}$ & $\mathrm{~A} \$ 25.20$ \\
\hline Cost of additional AI event & $\mathrm{A} \$ 40.20$ \\
\hline \multicolumn{2}{|l|}{ Genetic parameters } \\
\hline \multicolumn{2}{|l|}{ Estimated SD of breeding program $(\mathrm{BPI})^{3}$} \\
\hline AI-sired heifers & $\mathrm{A} \$ 90$ \\
\hline Non-AI-sired heifers & $\mathrm{A} \$ 45$ \\
\hline True SD of the population & $\mathrm{A} \$ 90$ \\
\hline Dam reliability, ${ }_{2}^{4} R_{d}$ & 0.32 \\
\hline Sire reliability, ${ }^{5} R_{s}$ & 0.66 \\
\hline Genomic reliability ${ }^{6} R_{G}$ & 0.66 \\
\hline Discount rate $^{7}$ & 0.08 \\
\hline \multicolumn{2}{|l|}{${ }^{1}$ From Byrne et al. (2016). } \\
\hline \multicolumn{2}{|c|}{$\begin{array}{l}{ }^{2} \text { This calculation assumed that a } 1 \text {-d delay to the onset of milking is worth } \mathrm{A} \$ 1.20 \text { per cow (T. J. Byrne, } \\
\text { AbacusBio, Edinburgh, Scotland, personal communication) and that animals that did not conceive on the first } \\
\text { AI event were eligible to be rebred for the remainder of the mating period. }\end{array}$} \\
\hline \multirow{2}{*}{\multicolumn{2}{|c|}{$\begin{array}{l}{ }^{3} \text { AI-sired heifers (G. J. Nieuwho } \\
\text { ers, a team of non-AI bulls are } \\
\text { The estimated SD of non-AI-sir } \\
\text { experts. BPI = Balanced Perfo } \\
{ }^{4} \text { Pryce et al. }(2010) \text {. }\end{array}$}} \\
\hline & \\
\hline \multicolumn{2}{|l|}{$\begin{array}{l}{ }^{5} \text { Sire reliability was cal } \\
{ }^{6} \text { Pryce et al. (2017). }\end{array}$} \\
\hline${ }^{7}$ McCullock et al. (2013), Partnerships Victoria (2003). & \\
\hline
\end{tabular}

4). This enabled us to account for the effect of fertility differences across age groups, in addition to the merit of using AI bulls, as opposed to non-AI bulls, as sires of calves. Values used for first-service conception rate in cows were taken from an Australian study by Morton (2011), and for heifers they were taken from Noonan et al. (2016), as they were not reported by Morton (2011).

In cows, we assumed an AI mating period of $9 \mathrm{wk}$, with 3 insemination opportunities; in heifers, we assumed that there was only one AI event, consistent with the practices of many Australian farms. Both heifers and cows were assumed to have a 6 -wk mating period (2 insemination opportunities) to a non-AI bull. Heat detection was assumed to be 0.8 (Byrne et al. 2016), with the exception of the first insemination in heifers, which was assumed to be part of a fixed-time AI program. Conception rates were assumed to be 5 percentage points lower at second service than at first service and 10 percentage points lower at third service for both cows and heifers. For example, a herd with a heifer first-service conception rate of 0.51 would have a second-service conception rate of 0.46 , and a third- service conception rate of 0.41 . Conception rates for cow fourth and fifth services (natural matings) were assumed to be 15 percentage points lower. Lower conception rates as the number of services increases is supported by field studies; for example, Healy et al. (2013) and DeJarnette et al. (2009).

Table 4. Summary of reproduction levels used in the 3 simulations

\begin{tabular}{|c|c|c|c|}
\hline \multirow[b]{2}{*}{ Reproduction parameter ${ }^{1}(\%)$} & \multicolumn{3}{|c|}{ Reproduction level } \\
\hline & Low & Medium & High \\
\hline Heifer first-service conception rate & 0.51 & 0.56 & 0.70 \\
\hline Total heifer in-calf ${ }^{2}$ rate & 0.79 & 0.84 & 0.93 \\
\hline Cow first-service conception rate & 0.36 & 0.43 & 0.49 \\
\hline Total cow in-calf rate from $\mathrm{AI}^{3}$ & 0.58 & 0.66 & 0.73 \\
\hline Total cow in-calf rate & 0.71 & 0.80 & 0.86 \\
\hline
\end{tabular}

${ }^{1}$ Values for cows' conception rate taken from Morton (2011); heifer conception rate taken from Noonan et al. (2016).

${ }^{2}$ In-calf rate is the total percentage of cows that are pregnant at the end of the mating period.

${ }^{3}$ The total percentage of cows that are pregnant to AI sires at the end of the mating period. 
Sexed Semen. Sexed semen was assumed to be used for the first insemination of both heifers and cows, in line with current industry practice in Australia. When inseminations were successful, we assumed that $90 \%$ of calves born were female (Butler et al., 2014). A decrease in conception rate varying between 0 and 30 percentage points was modeled for inseminations with sexed semen, representative of published results (i.e., Noonan et al., 2016; Table 2). For example, a 10-percentagepoint decrease in conception rate with sexed semen would reduce first-service conception rate from 0.51 to 0.41 . When a decrease in sexed semen conception rate occurred, both the calving pattern of the herd and the number of cows pregnant at the end of the mating period changed. Scenarios that were equivalent except for their sexed semen usage were used to compare the number of females pregnant at 21-d intervals throughout the mating period. We assumed a full cycle passed before a female had another opportunity to conceive. For example, in a herd with low reproductive performance and where using sexed semen results in a 5-percentage-point decrease in first-service conception rate, there were 1.7 fewer cows pregnant at the end of the mating period (i.e., 69 cows pregnant compared with 70.7 cows pregnant in an equivalent herd using only conventional semen). Additionally, in this example, the effect of a 5-percentage-point decrease in first service rate is to delay 13 calving dates by $21 \mathrm{~d}$, compared with a scenario with equivalent reproductive performance.

Two approaches were taken in the evaluation of sexed semen usage in conjunction with genomic testing. In scenario 2, the only potential benefit considered was the effect on genetic gain, where the only additional cost included, aside from the cost of genomic testing, was purchasing sexed semen (Table 1). Scenario 3 included other costs, losses, and benefits associated with adoption of sexed semen including (1) MRH, (2) lost income due to fewer male calf sales, (3) cost of additional inseminations in cows that do not conceive to sexed semen, and (4) lost milk income (Tables 2 and 3).

A partial budget approach was used to capture the changes in income and expenditure associated with the use of sexed semen and genomic testing. This approach means that only changes in income or expenditure directly associated with, or altered by, the use of sexed semen are included. For example, straws of sexed semen cost $\mathrm{A} \$ 50$ on average, compared with $\mathrm{A} \$ 20$ for conventional semen (Byrne et al., 2016). Using sexed semen at the first insemination replaces a straw of conventional semen, so a value of $\mathrm{A} \$ 30$ representing the cost of this change would be multiplied by the number of animals inseminated with sexed semen. A reduction in first-service conception rate delays the calving date for a portion of the herd. This increases AI costs in cows because additional cows require a second round of AI compared with a scenario with no sexed semen. Taking a partial budget approach means that the cost of conventional semen is only considered when additional semen is used. A delay to calving date also leads to lost milk income, as increased calving interval reduces overall milk yield. Byrne et al. (2016) describes a series of formulas for calculating the value of a decrease in average total milk yield, per day per cow, for every $1-d$ extension to the calving interval. The cost of a 1-d delay to calving of $\$ 1.20$ per cow per day (T. J. Byrne, AbacusBio, Edinburgh, Scotland, UK, personal communication) was used. We assumed that animals that did not conceive on first service with sexed semen were eligible to be remated every $21 \mathrm{~d}$ for the remainder of the mating period, giving a total value of lost milk of $\mathrm{A} \$ 25.20$ per female per failed mating as a result of using sexed semen at the first insemination.

Following extensive consultation with industry experts on rearing costs and sale prices and consultation of industry resources (i.e., Dairy Australia 2013), a range of $\mathrm{A} \$ 0$ to $\mathrm{A} \$ 600$ for $\mathrm{MRH}$ was chosen for this study (Table 2).

Selection Strategy. Two selection strategies were considered. In the first instance, herd replacement rate was assumed to be equal to involuntary cull rate (mortality rate plus the number of cows not pregnant at the end of the mating period). In the second strategy, higher replacement rates, which allowed voluntary culling of cows, were considered. Herein we report results (1) where the replacement rate is equal to the involuntary cull rate, and (2) the replacement rate where cost of genomic testing was equal to the additional merit expressed by selected heifers over their lifetime.

Heifers born from AI and natural matings were eligible for selection as replacements. The sole selection criterion used to select replacements was the Balanced Performance Index (BPI). The BPI is Australia's main selection index and includes EBV for production, health, fertility, conformation, survival, and feed saved. A 1-unit increase in BPI is expected to reflect an increase of $\mathrm{A} \$ 1$ in net farm profit per cow per year (refer to Byrne et al., 2016 for further details). Heifer selection candidates were assumed to come from 2 populations: AI heifers and offspring of natural matings. The genetic merit of each population was assumed to follow a normal distribution of BPI. We assumed the true standard deviation (SD) of BPI was $\mathrm{A} \$ 90$ in both populations (G. J. Niewwhof, DataGene, Melbourne, Victoria, Australia, personal communication), and that the SD of the EBV for BPI in 2 calf populations would differ. The SD of the EBV for BPI for AI-born calves was assumed to be $\mathrm{A} \$ 90$, whereas for calves from nonAI bulls, the SD of EBV for BPI was assumed to be 
$\mathrm{A} \$ 45$. The true SD of BPI is approximately the same as the SD of EBV of BPI in calves sired by AI, as the population of AI sires includes overlapping generations. In contrast, the bulls used in non-AI matings would typically be a discrete generation and thus the SD of EBV would be lower. The mean BPI of AI born calves was assumed to be $\mathrm{A} \$ 0$, whereas for daughters of nonAI bulls, the mean was assumed to be $-\mathrm{A} \$ 62.70$ (Byrne et al., 2016), which is the difference in average genetic merit of calves born from herd-bull matings compared with AI matings. Although, on average, AI-born calves are of greater genetic merit than calves from non-AI herd bull matings, at the individual level, some of these calves will have greater genetic merit than AI calves, as illustrated in Figure 1. To account for this, selection of heifer replacements was based on truncation selection of the BPI using EBV across natural and AI-born calf populations. The methodology of Hopkins and James (1978) was followed to find the BPI value that resulted in the desired number of herd replacements being selected from the 2 populations. This is represented by a vertical line in Figure 1. An optimization equation iterated over BPI values of the calf population to find the BPI that resulted in the desired number of herd replacements. As the selection intensity, $i_{\text {heifers }}$, in the 2 calf populations differed, $i_{\text {heiffers }}$ was calculated separately for each population. A weighted average was then calculated based on the number of selected heifers that came from each population, for use in subsequent calculations. Similarly, a weighted average was calculated for the truncation point of the standard normal distribution of BPI corresponding to the selected proportion of animals in each population, $x_{\text {heifers }}$.

Calculation of Reliability. The reliability of genomic testing $\left(\boldsymbol{R}_{\boldsymbol{G}}\right)$ was fixed at 0.66 based on the current reliability of genomic test results for BPI (Pryce et al., 2017). To calculate the reliability of $\mathrm{EBV}_{\mathrm{PA}}$, dams were assumed not to have genomic information, so the reliability of the dam's EBV, $R_{d}$, was fixed at 0.32 (Pryce et al., 2010; Table 3). Sire reliability, $R_{s}$, was assumed to be 0.66 , which includes an adjustment for a pedigree error rate of $10 \%$ using methodology of Visscher et al. (2002).

Bijma (2012) demonstrated that the reliability of an $\mathrm{EBV}_{\mathrm{PA}}$ and reliability of selection based on $\mathrm{EBV}_{\mathrm{PA}}$ are not the same in selected populations. The formula of Bijma (2012) was used to derive the reliability of selection based on $\mathrm{EBV}_{\mathrm{PA}}, R_{P A}$;

$$
\begin{aligned}
R_{P A} & =\frac{1}{4}\left(1-k_{s}\right) R_{s}+\frac{1}{4}\left(1-k_{d}\right) R_{d}, \\
k_{s} & =i_{s}\left(i_{s}-x_{s}\right), \\
k_{d} & =i_{d}\left(i_{d}-x_{d}\right),
\end{aligned}
$$

where sire and dam are denoted by subscripts $s$ and $d$, respectively, $k$ is the proportional reduction in variance of the selection criterion, $i$ is the selection intensity, and $x$ represents the truncation point of the standard normal distribution of BPI corresponding to the selected proportion of animals. For sires, we assumed that $2 \%$ of sires were selected, so $i_{s}=2.42$. Selection intensity was assumed to be the same each year in the scenario, so $i_{d}=i_{\text {heifers }}$, and $x_{d}=x_{\text {heifers. }}$. The reliability of $\mathrm{EBV}_{\mathrm{PA}}$ was 0.25, whereas after Bijma's (2012) correction, the reliability of selection based on $\mathrm{EBV}_{\mathrm{PA}}$ was lower, at $\sim 0.06$.

\section{Calculating Net Benefit}

Genomic Selection. The benefits of genomic selection were assumed realized only through the ability to make more accurate decisions about which heifers to keep. This can be represented by an adaption of the breeder's equation (e.g., Falconer, 1989):

$$
\text { Benefit }=\sigma_{B P I} \times i_{\text {heifers }} \times\left(\sqrt{R_{G}}-\sqrt{R_{c}}\right),
$$

where $\sigma_{B P I}$ is the true SD of $\mathrm{BPI}(=\mathrm{A} \$ 90)$, and $R_{c}$ refers to the reliability of the current selection strategy.

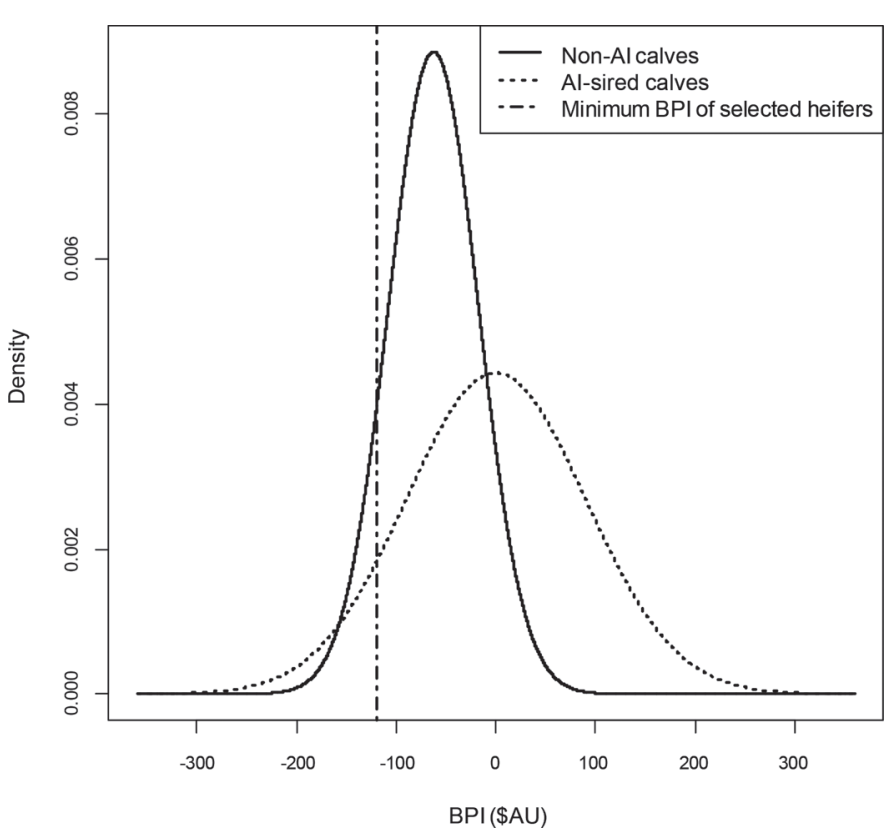

Figure 1. Example distribution of the Balanced Performance Index (BPI, $\mathrm{A} \$$ ) for calves sired by $\mathrm{AI}$ and non-AI matings. The vertical line represents an example truncation point- the minimum BPI for a heifer to be retained as a replacement-illustrating that both calves from AI matings and naturally sired calves meet this threshold. This truncation point will change depending on the number of heifers that are required as replacements. 
When current selection decisions are based on $\mathrm{EBV}_{\mathrm{PA}}$, $R_{c}=R_{P A}$, and when no $\mathrm{EBV}_{\mathrm{PA}}$ are available, $R_{c}=0$. The average merit of a heifer selected as a herd replacement over all heifer selection candidates was assumed to be expressed once the heifer entered the milking herd, and then for every lactation she remained in the herd. Changes to replacement rate also affect the average length of time that heifers stay in the herd. The net present value of heifer merit was calculated based on the average productive life, $\mathrm{PL}=1 /$ replacement rate, of the herd.

Sexed Semen. The calculation of net benefit from genotyping and using sexed semen also included the costs of sexed semen, lost income with fewer male calves sold, additional heifer rearing costs, and surplus heifer sales. When a reduction in conception rate occurred, lost milk income due to a delay in the start of milking and additional AI costs were also accounted for. Costs that occurred before genomic testing (i.e., sexed semen and additional AI costs) were inflated, and costs that occurred at a similar time to genomic testing were not discounted.

Thus, net benefit from genotyping with or without using sexed semen over the lifetime of the selected heifers (in $\mathrm{A} \$ / 100$ cows) can be expressed as follows:

$$
\begin{aligned}
& \text { Net benefit }= \\
& n_{\text {heifers }} \times \sum_{t=3}^{P L}\left(\frac{\sigma_{B P I} \times i_{\text {heifers }} \times\left(\sqrt{R_{G}}-\sqrt{R_{c}}\right)}{(1+d r)^{t}}\right) \\
& -C_{\text {genotyping }}+C_{S S A},
\end{aligned}
$$

where $n_{\text {heifers }}$ is the total number of heifers selected, $t$ is the time in years since heifers' birth, $P L$ is the productive life, $d r$ is the discount rate of $8 \%$, and $C_{\text {genotyping }}$ is the net cost of genotyping all heifer selection candidates. In applying the discount rate, genomic testing was assumed to be time zero. Finally, $C_{S S A}$ is the adjustment for additional costs and losses associated with sexed semen. For scenarios with no sexed semen usage, this value was $\mathrm{A} \$ 0$.

\section{RESULTS}

\section{Scenario 1: Adoption of Genomic Testing Only}

All parameters varied in scenario 1 had an effect on the net benefit of genomic testing in $\mathrm{A} \$$ per 100 cows, although the magnitude of this effect differed between parameters (Table 5). Across the range of values tested, it was hard to determine the largest effect due to different units for each parameter. However, herd reproductive performance and the prior availability of $\mathrm{EBV}_{\mathrm{PA}}$ had very large effects on net benefit. The cost of genomic testing had a smaller influence. It was assumed that a herd's replacement rate must be equal to or greater than the number of cows that exited the herd each year (i.e., cow deaths and cows not pregnant at the end of the mating period).

Herd Reproduction Rate and Replacement Rate. Herd reproduction rate, in particular the number of nonpregnant cows at the end of the mating period, had a large effect on the number of herd replacements. For herds with low reproductive performance, the net

Table 5. Net benefit (NB, A $\$ / 100$ cows) from genotyping heifers in herds with differing reproduction (repro) levels with and without parentderived $\mathrm{EBV}\left(\mathrm{EBV}_{\mathrm{PA}}\right)$ available, assuming a genomic test reliability of $0.66^{1}$

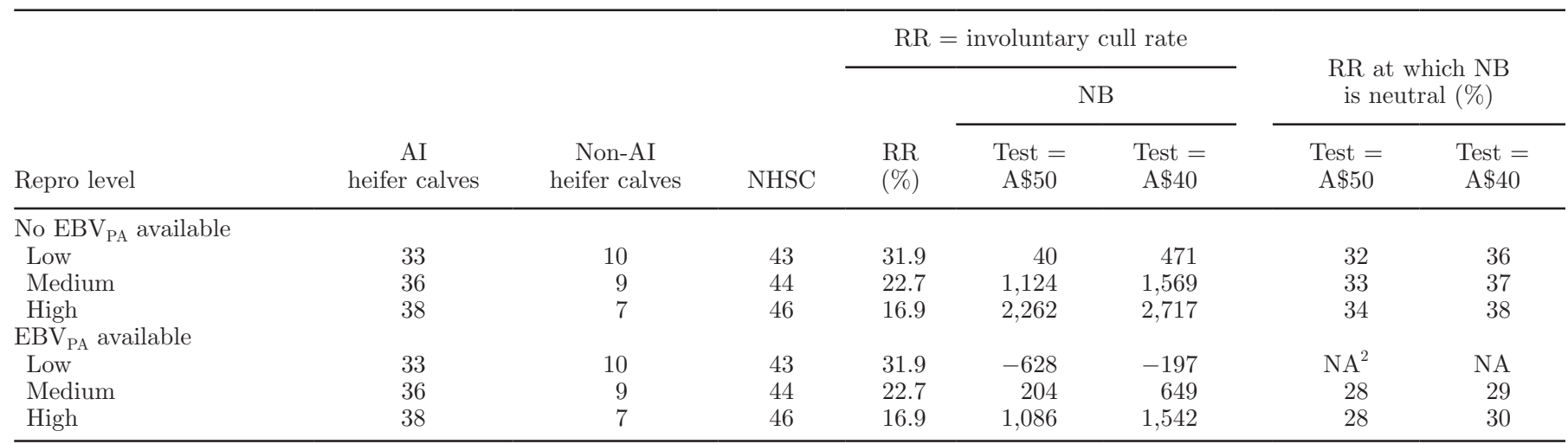

${ }^{1} \mathrm{~A}$ neutral NB is defined as the replacement rate (to the nearest percentage) at which the cost of genomic testing is approximately equal to the contribution that heifers chosen as replacements make to farm profit over their lifetime. Two different genomic test costs (A $\$ 40$ and $\mathrm{A} \$ 50 /$ heifer) were considered. The number of heifer calves born from AI and non-AI matings and the total number of selection candidates (NHSC) are also given. Two outcomes are presented here: (1) NB where replacement rate (RR, \%) is assumed to equal involuntary cull rate, and (2) RR where NB is neutral.

${ }^{2} \mathrm{NA}$ indicates none of the replacement rates tested had a positive net benefit. 
Table 6. Net benefit (NB, $\$ \mathrm{~A} / 100$ cows) from genotyping commercial heifers in herds with differing reproduction (repro) levels with and without parent-derived $\mathrm{EBV}\left(\mathrm{EBV}_{\mathrm{PA}}\right)$ available, assuming a genomic test reliability of $0.76^{1}$

\begin{tabular}{|c|c|c|c|c|c|c|c|c|}
\hline \multirow[b]{2}{*}{ Repro level } & \multirow[b]{2}{*}{$\begin{array}{c}\text { AI } \\
\text { heifer calves }\end{array}$} & \multirow[b]{2}{*}{$\begin{array}{l}\text { Non-AI } \\
\text { heifer calves }\end{array}$} & \multirow[b]{2}{*}{ NHSC } & \multicolumn{3}{|c|}{ NB $(\mathrm{A} \$ / 100$ cows $)$} & \multicolumn{2}{|c|}{$\begin{array}{c}R R \text { at which NB is neutral } \\
(\%)\end{array}$} \\
\hline & & & & $\mathrm{RR}$ & $\begin{array}{l}\text { Test }= \\
\$ A 50\end{array}$ & $\begin{array}{l}\text { Test }= \\
\$ A 40\end{array}$ & $\begin{array}{l}\text { Test }= \\
\$ \mathrm{~A} 50\end{array}$ & $\begin{array}{l}\text { Test }= \\
\$ \mathrm{~A} 40\end{array}$ \\
\hline \multicolumn{9}{|c|}{ No $\mathrm{EBV}_{\mathrm{PA}}$ available } \\
\hline Low & 33 & 10 & 43 & 31.9 & 200 & 632 & 34.0 & 37.0 \\
\hline Medium & 36 & 9 & 44 & 22.7 & 1,369 & 1,813 & 35.0 & 38.0 \\
\hline \multicolumn{9}{|c|}{$\mathrm{EBV}_{\mathrm{P}}$ available } \\
\hline Low & 33 & 10 & 43 & 31.9 & -467 & -36 & $\mathrm{NA}^{2}$ & NA \\
\hline Medium & 36 & 9 & 44 & 22.7 & 449 & 893 & 29.0 & 32.0 \\
\hline High & 38 & 7 & 46 & 16.9 & 1,418 & 1,873 & 29.0 & 33.0 \\
\hline
\end{tabular}

${ }^{1} \mathrm{~A}$ neutral NB is defined as the replacement rate (to the nearest percentage) where the cost of genomic testing is approximately equal to the contribution that heifers chosen as replacements make to farm profit over their lifetime. Two genomic test costs (A $\$ 40$ and A $\$ 50 /$ heifer) were considered. The number of heifer calves born from AI and non-AI matings and the total number of selection candidates (NHSC) are also given. Two outcomes are presented here: (1) NB where replacement rate (RR, \%) is assumed to equal involuntary cull rate, and (2) RR where NB is neutral

${ }^{2} \mathrm{NA}$ indicates none of the replacement rates tested had a positive net benefit.

benefit of genomic testing was never positive when $\mathrm{EBV}_{\mathrm{PA}}$ were already available. Overall, the net benefit of genomic testing increased as reproduction rate increased, primarily due to lower replacement rates. When reproductive performance was medium or high, there was a difference in the minimum replacement rate needed to sustain herd size and the maximum number of replacements that could be retained and still recoup the investment in genomic testing. For example, for a herd with medium reproductive performance, replacement rate ranged from a minimum of $22.7 \%$ to a neutral benefit at $33 \%$ when no $\mathrm{EBV}_{\mathrm{PA}}$ where available (Table $5)$. We consider the net benefit to be neutral when the cost of genomic testing is approximately equal to the contribution that heifers chosen as replacements make to farm profit over their lifetime.

Availability of $\boldsymbol{E} \boldsymbol{B} \boldsymbol{V}_{\boldsymbol{P A}}$. The net benefit from genomic testing was greater when $\mathrm{EBV}_{\mathrm{PA}}$ were not available on selection candidates compared with when $\mathrm{EPV}_{\mathrm{PA}}$ were available. For example, net benefit was $\mathrm{A} \$ 920$ lower per 100 cows when comparing 2 simulations with and without $\mathrm{EPV}_{\mathrm{PA}}$ for a herd with medium reproduction rate. The point at which the net benefit of genomic testing was neutral occurred at replacement rates of 5 to 6 percentage points higher when $\mathrm{EBV}_{\mathrm{PA}}$ were not available (Table 5).

Cost and Reliability of Genomic Test. A decrease of $\mathrm{A} \$ 1$ in the cost of genomic testing increased the net benefit from genomic testing by $\mathrm{A} \$ 44$ per 100 cows, on average. For example, when a medium reproduction rate and genomic test cost of $\mathrm{A} \$ 50$ were used, the net benefit was $\mathrm{A} \$ 1,124$ per 100 cows, whereas with a genomic test cost of $\mathrm{A} \$ 40$, net benefit was $\mathrm{A} \$ 1,569$, assuming $\mathrm{EPV}_{\mathrm{PA}}$ were unavailable. As the genomic test cost decreased, the replacement rate at which the net benefit was neutral increased. For example, when no $\mathrm{EPV}_{\mathrm{PA}}$ were available, the net benefit of genomic testing was neutral at replacement rates that were $4 \%$ higher when the genomic test cost decreased from $\mathrm{A} \$ 50$ to A $\$ 40$ (Table 5).

If a genomic test reliability of 0.76 was considered, the net benefit in $\mathrm{A} \$$ per 100 cows was higher than the same scenario with reliability of 0.66 . The replacement rate at which net benefit was neutral was 1 to 2 percentage points higher than at genomic test reliabilities of 0.66 (Table 6).

\section{Scenario 2: Cost of Using Sexed Semen Only}

Net benefit always decreased when sexed semen and genomic testing were used concurrently, when the benefit of sexed semen (compared with no usage) was limited to the effect on selection intensity and the only costs considered were the direct costs of genomic testing and purchasing sexed semen (scenario 2, results not shown). The expected decrease in net benefit was even greater when a reduction in conception rate in females mated to sexed semen was assumed to occur. So, the extra genetic gain from having more surplus heifers did not compensate for the additional costs of sexed semen and genotyping more selection candidates. This was also true at a genomic reliability of 0.86 .

\section{Scenario 3: Considering All Costs, Losses, and Benefits of Using Sexed Semen}

When other costs, losses, and benefits associated with using sexed semen were included in net benefit 
Table 7. The net benefit (A $\$ / 100$ cows) from using sexed semen (SS) in conjunction with genomic testing compared with the equivalent scenario with genomic testing only in a herds with different levels of reproductive performance $(\mathrm{RP})^{1}$

\begin{tabular}{|c|c|c|c|c|c|c|c|c|c|}
\hline \multirow{2}{*}{ MRH (A\$/heifer) } & \multirow{2}{*}{$\begin{array}{l}\text { Bred to } \\
\text { SS (\%) }\end{array}$} & \multicolumn{4}{|c|}{ SS in heifers $(\mathrm{A} \$ / 100$ cows $)$} & \multicolumn{4}{|c|}{ SS in cows $(\mathrm{A} \$ / 100$ cows $)$} \\
\hline & & \multicolumn{4}{|c|}{ Decrease in CR (percentage points) } & \multicolumn{4}{|c|}{ Decrease in CR (percentage points) } \\
\hline \multicolumn{10}{|l|}{ Low RP } \\
\hline \multirow[t]{2}{*}{0} & 50 & -518 & -864 & $-1,225$ & $-1,586$ & $-1,903$ & $-2,456$ & -3041 & $-3,664$ \\
\hline & 100 & $-1,057$ & $-1,460$ & $-1,876$ & $-2,282$ & $-3,900$ & $-4,655$ & $-5,498$ & $-6,432$ \\
\hline \multirow[t]{2}{*}{400} & 50 & 565 & 56 & -467 & -990 & 17 & -940 & $-1,929$ & $-2,956$ \\
\hline & 100 & 1,109 & 381 & -360 & $-1,091$ & -60 & $-1,624$ & $-3,275$ & $-5,016$ \\
\hline \multirow[t]{2}{*}{600} & 50 & 1,107 & 516 & -88 & -692 & 977 & -182 & $-1,373$ & $-2,602$ \\
\hline & 100 & 2,192 & 1,301 & 398 & -495 & 1,860 & -108 & $-2,163$ & $-4,308$ \\
\hline \multicolumn{10}{|l|}{ Medium RP } \\
\hline \multirow[t]{2}{*}{0} & 50 & -433 & -759 & $-1,084$ & $-1,409$ & $-2,116$ & $-2,560$ & $-3,016$ & $-3,475$ \\
\hline & 100 & -875 & $-1,236$ & $-1,597$ & $-1,952$ & $-4,269$ & $-4,849$ & $-5,455$ & $-6,084$ \\
\hline 600 & 100 & 1,671 & 976 & 282 & -406 & 2,611 & 922 & -792 & $-2,529$ \\
\hline \multicolumn{10}{|l|}{ High RP } \\
\hline \multirow[t]{2}{*}{0} & 50 & -332 & -638 & -945 & $-1,269$ & $-2,151$ & $-2,535$ & $-2,949$ & $-4,029$ \\
\hline & 100 & -658 & $-1,006$ & $-1,354$ & $-1,684$ & $-4,376$ & $-4,842$ & $-6,067$ & $-6,599$ \\
\hline \multirow[t]{2}{*}{200} & 50 & 62 & -282 & -625 & -986 & -845 & $-1,401$ & $-1,988$ & $-3,240$ \\
\hline & 100 & 129 & -293 & -715 & $-1,118$ & $-1,763$ & $-2,574$ & $-4,145$ & $-5,022$ \\
\hline \multirow[t]{2}{*}{400} & 50 & 455 & 75 & -306 & -703 & 462 & -267 & $-1,026$ & $-2,452$ \\
\hline & 100 & 916 & 421 & -75 & -552 & 850 & -306 & $-2,222$ & $-3,445$ \\
\hline \multirow[t]{2}{*}{600} & 50 & 849 & 432 & 14 & -420 & 1.769 & 867 & -65 & -1.663 \\
\hline & 100 & 1,703 & 1,134 & 565 & 13 & 3,464 & 1962 & -300 & $-1,868$ \\
\hline
\end{tabular}

${ }^{1}$ Results are displayed as the net benefit from using SS minus the net benefit of genomic testing only in equivalent scenarios. Parameters varied included using SS in a variable proportion (50 or 100\%) of heifers or cows, the decrease in conception rate (CR, 0 to 15 percentage points) in females mated to SS, and the marginal return for surplus heifers ( $\mathrm{MRH}, \mathrm{A} \$ 0$ to $\mathrm{A} \$ 600$ per heifer). We assumed that parent-derived EBV (EBV $\mathrm{PA}_{\text {) }}$ were not available, a genomic test cost $\mathrm{A} \$ 50$ per heifer, and genomic reliability was 0.66 .

calculations (scenario 3), using sexed semen with genomic testing had a highly variable effect on net benefit (Table 7). The results presented are based on a genomic test cost of $\mathrm{A} \$ 50 /$ heifer, no $\mathrm{EBV}_{\mathrm{PA}}$ availability, $\mathrm{EBV}_{\mathrm{G}}$ reliability of 0.66 , and a herd replacement rate equal to the herd culling rate (Table 7). Scenario 3 was more likely to have a higher net benefit than the equivalent scenario without sexed semen if (1) MRH was at least $\$ 400,(2)$ sexed semen was used in heifers, and (3) reductions in conception rate associated with sexed semen usage were $10 \%$ or less. Although genomic test cost influenced the overall net benefit realized, it had only a very small effect on which combinations of variables yielded a net benefit greater than the equivalent scenario without sexed semen. Results from a genomic test cost of $\mathrm{A} \$ 40$ are not included here.

Marginal Return for Surplus Heifers. Marginal return for surplus heifers had a large impact on whether using sexed semen resulted in a larger net benefit than genomic testing alone. A positive net benefit (compared with the equivalent scenario with no sexed semen) was most likely to occur when MRH was $\mathrm{A} \$ 400$ or more. When MRH was A $\$ 200$ or less and sexed semen was used in cows, the net benefit never increased (compared with the equivalent scenario with no sexed semen; Table 7). When MRH was A $\$ 200$, small increases in net benefit, up to $\$ 129$ per 100 cows, were seen when sexed semen was used in heifers in simulations with low or high reproduction rate, as long as no reduction in conception rate occurred.

Sexed Semen in Heifers Versus Cows. Net benefit increased more for every heifer inseminated with sexed semen than for every cow. In our study, net benefit increased by up to $\mathrm{A} \$ 101$ per heifer inseminated with sexed semen compared with up to $\mathrm{A} \$ 35$ per cow inseminated with sexed semen. Where net benefit was positive, the overall increase in net benefit per 100 cows tended to be greater when sexed semen was used in heifers than in cows, despite there being fewer heifers than cows in a herd. The exception to this was when $\mathrm{MRH}$ was $\mathrm{A} \$ 600$ and reproductive performance was high. Overall, there were more scenarios where net 
Table 8. Overview of the change in herd replacement rate (RR, \%) and number of heifer selection candidates (NHSC) when sexed semen (SS) is used in conjunction with genomic testing across 3 levels of reproductive performance $(\mathrm{RP})^{1}$

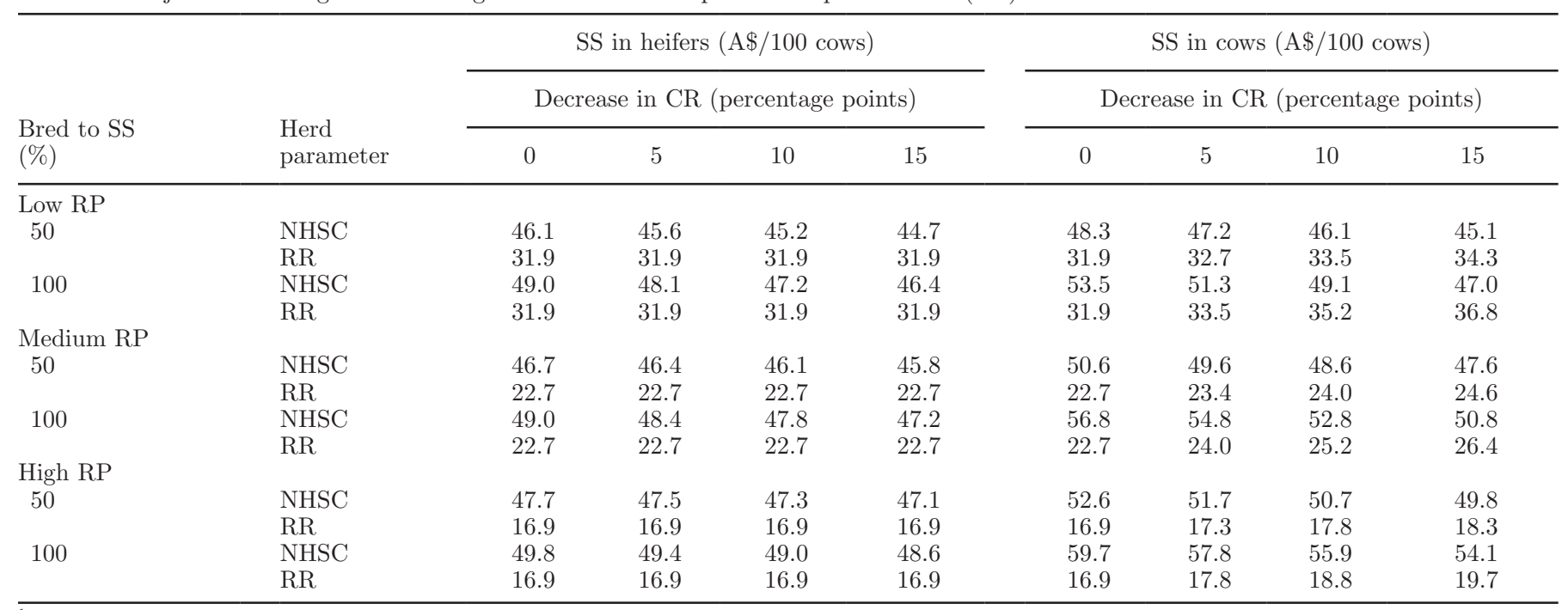

${ }^{1}$ Parameters varied included using SS in a variable proportion of heifers or cows (50 or 100\%) and the decrease in conception rate (CR, 0 to 15 percentage points) in females bred to SS. We assumed parent-derived $\mathrm{EBV}\left(\mathrm{EBV}_{\mathrm{PA}}\right)$ were not available.

${ }^{2}$ The proportion of heifers that are required as selection candidates can be calculated as RR/NHSC, where RR is equivalent to the number of heifers that are needed to replace culled cows and NHSC is the number of available heifers.

benefit increased when sexed semen was used in heifers only as opposed to in cows. Table 7 shows 32 scenarios where using sexed semen in heifers increased net benefit (compared with same scenarios without sexed semen usage). In contrast, using sexed semen in cows only increased the net benefit for 15 of the scenarios tested across the same range of parameters. If inseminating $25 \%$ of heifers with sexed semen was profitable, mating all heifers to sexed semen under the same herd parameters was also profitable. The same was found in cows. The exception to this was that it is not profitable to use sexed semen in all cows when $\mathrm{MRH}$ was $\mathrm{A} \$ 400$ and herd reproduction rate was low. Only a small number of scenarios that used sexed semen in both heifers and cows had a higher net benefit that using sexed semen in heifers alone. They only occurred when MRH was $\mathrm{A} \$ 400$ or greater and the decrease in conception rate was no more than 5 percentage points (results not shown).

Decreased Conception Rate when Sexed Semen Is Used. When using sexed semen led to a reduction in conception rate of 15 percentage points, or less, there were instances where the net benefit increased (compared with the equivalent scenario without sexed semen; Table 7). Where using sexed semen decreased conception rates, the number of heifer selection candidates available was reduced (Table 8 ), although the number of selection candidates remained higher than the equivalent scenario with no sexed semen usage
(Table 5). The impact of decreases in conception rate on net benefit was different in cows and heifers and for different reproduction levels. For example, when sexed semen was used in cows, net benefit never increased if a reduction in conception rate greater than 5 percentage points occurred. In contrast, net benefit still increased if sexed semen was used in heifers with decreases in conception rate of up to 15 percentage points. However, decreases in heifer sexed semen conception rate of 10 percentage points or more could only be justified when MRH was A $\$ 600$ or higher. Similarly, MRH of at least $\mathrm{A} \$ 600$ was needed to compensate for a 5-percentagepoint decrease in conception rate in cows (Table 7).

Herd Reproduction. Net benefit was highest when sexed semen was used and herd reproduction rate was high (Table 7). This is partly because the effect of a decrease in conception rate was lower for high reproduction scenarios (Table 8). For example, when all heifers were inseminated with sexed semen and a 15-percentage-point decrease in conception rates occurred, there were 2.6 (i.e., 49.0 minus 46.4) fewer heifer selection candidates in a low reproduction scenario. In contrast, there were only 1.2 fewer heifer selection candidates in a high reproduction scenario (Table 8). Overall, the percentage increase in net benefit (compared with the equivalent scenario without sexed semen) when sexed semen was used was greatest with low herd reproduction rates. This can be illustrated by comparing the difference in using sexed semen in all cows and heifers 
compared with none across the reproduction levels, assuming a genomic test cost of $\mathrm{A} \$ 50$ and a heifer return of $\mathrm{A} \$ 400$. At a low reproduction level, using sexed semen increased net benefit by $\mathrm{A} \$ 1,109$ (from $\mathrm{A} \$ 40$ to $\mathrm{A} \$ 1,149)$, representing a 29 -fold increase in net benefit. At high herd reproduction levels, using sexed semen increased net benefit by $\mathrm{A} \$ 916$ (from $\mathrm{A} \$ 2,262$ to $\mathrm{A} \$ 3,178)$, which only represents a $50 \%$ increase in net benefit.

\section{DISCUSSION}

In this study, we considered several herd parameters that influence the net benefit ( $\mathrm{A} \$$ per 100 cows) from using genomic selection alone or together with sexed semen. The investment made in genotyping heifer calves to more accurately select herd replacements can be recouped within the lifetime of the animal. At reliabilities and replacement rates representative of the current averages in Australia $\left(R_{G}=0.66\right.$, Pryce et al., 2017; replacement rate $=22.5 \%$, Byrne et al., 2016), this ranged from $\mathrm{A} \$ 204$ to $\mathrm{A} \$ 1,124$ per 100 cows depending on whether $\mathrm{EBV}_{\mathrm{PA}}$ were or were not available. As replacement rates increase, the net benefit becomes smaller, becoming unprofitable at replacement rates of $28 \%$ ( $\mathrm{BV}_{\mathrm{PA}}$ available) and $33 \%$ (no $\mathrm{EBV}_{\mathrm{PA}}$ available) for an average dairy herd under current parameters. When sexed semen is used to increase the number of heifer calves and all calves genotyped, the net benefit varies greatly and is highly influenced by the MRH. A positive net benefit, greater than genomic testing alone, is most likely to be seen when sexed semen is used in heifers, conception rates do not decrease by more than 10 percentage points below those of conventional semen and MRH is greater than $\$ 400$.

\section{Genomic Testing to Guide Replacement Decisions}

Although the number of females being commercially genomic tested is rapidly increasing, the overall percentage of the total population of young heifers that is genotyped is still relatively small in many countries. This study has demonstrated that, even at current costs and reliabilities, genomic testing as a guide for heifer replacement decisions only is profitable for many Australian farmers. Although the parameters chosen here are reflective of Australian systems, results are likely to apply to pasture-based dairy industries in other parts of the world (e.g., New Zealand and Ireland). The principles used in this study can be adapted so that the calculations represent other dairy systems.

In herds with moderate to high reproductive performance, an opportunity exists to keep additional heifer replacements, compared with only replacing involuntary cull cows (deaths and infertility). This provides an opportunity for voluntary culling; that is, to cull poorer performing cows, such as those with low production or chronic high cell count. Decreases in genomic test cost will increase the scope for voluntary culling including herds with lower reproductive performance. Continued increases in genomic reliabilities will increase the number of herds for which voluntary culling may be a profitable option. For example, if genomic reliabilities increase to 0.76 , herds with low reproductive performance without access to $\mathrm{EBV}_{\mathrm{PA}}$ will now have a small window for voluntarily culling. Increasing replacement rate decreases the average age of the herd. Although this has a positive effect on rate of genetic gain, it also has implications for farm profitability, as cows generally do not reach their peak milk production until after 4 yr of age (i.e., Byrne et al., 2016). Such considerations are outside the scope of this paper.

Unsurprisingly, we found that the net benefit from genomic testing was lower when farmers already have access to $\mathrm{EBV}_{\mathrm{PA}}$ to make selection decisions. This supports the conclusions of others (i.e., Weigel et al., 2012; Calus et al., 2015; Pryce and Hayes, 2012). Although Calus et al. (2015) accounted for the effect of reliability in selected populations and accrued benefits over cow lifetimes in their calculations, the estimates in our study tend to be more conservative than those of Calus et al. (2015). This can be partially attributed to including discounting over time in our study. The benefits of genomic testing are not typically realized at the time of testing but instead are expressed by selected individuals for each lactation in their productive life. A heifer with a BPI $\mathrm{A} \$ 10$ greater than her contemporaries and a productive life of $4 \mathrm{yr}$ is expected to contribute $\mathrm{A} \$ 40$ more to farm profit over her lifetime without applying discounting (a 1-unit increase in BPI equates to a $\mathrm{A} \$ 1$ / cow per year increase in profit). If a discount rate of $8 \%$ is used, her lifetime contribution to profit is almost $30 \%$ lower at $\mathrm{A} \$ 28.40$. A discount rate of $8 \%$, as used in this study, could be considered high; however, it aligns with discount rates recommended by Australian state governments (i.e., Victorian Department of Treasury and Finance; Partnerships Victoria 2003) and the discount rate used by McCullock et al. (2013). Thus, the predicted net benefit in this study is a more conservative estimate of the net benefits of genomic testing.

\section{Adoption of Sexed Semen and Genomic Testing}

An opportunity also exists to realize potentially greater benefits from genomic selection by using it in tandem with female reproductive technologies. Many 
female reproductive technologies that were previously deemed unprofitable may now have a place in breeding programs (i.e., Boichard et al., 2013; Granleese et al., 2015), provided pregnancy rates are high (i.e., Fetrow et al., 2007; Cabrera, 2009). Our results show that conception rates from using sexed semen need to be similar to those with conventional semen for concurrent adoption of sexed semen and genomic testing to generate a greater net benefit than genomic testing alone. A study by Fetrow et al. (2007) concluded that sexed semen is likely to be less profitable for cows than for heifers, which was also supported by our study. If the net benefit of using sexed semen in addition to genomic testing is considered in terms of $\mathrm{A} \$$ per female inseminated with sexed semen instead of $\mathrm{A} \$$ per 100 cows, the net benefit increases more for every heifer inseminated with sexed semen than for every cow. In our study, this ranged up to $A \$ 101$ per heifer inseminated with sexed semen compared with a maximum of $\mathrm{A} \$ 35$ per cow inseminated with sexed semen. With their inherently higher fertility level, heifers are more likely to conceive at first service (Morton, 2011; Noonan et al., 2016). This better balances the lower fertility and higher cost of sexed semen, hence the industry recommendations of sexed semen usage in heifers in preference to cows (Izzo 2015). Industry confidence in using sexed semen in heifers should be lifted by (1) recent increases in sexed semen fertility (Butler et al., 2014), and (2) our demonstration that using sexed semen in heifers and genomic testing can generate a greater net benefit than genomic testing alone, even with up to a 10-percentagepoint reduction in conception rate.

Relying on the additional genetic gain from concurrent adoption of genomic testing and using sexed semen is not enough to recoup the initial investment in genomic testing and sexed semen, as demonstrated in scenario 2. This is still true at genomic test reliabilities of 0.86 (results not displayed) and elsewhere (Boichard et al., 2013). As in our study, Boichard et al. (2013) accrued genetic gain in selected heifers over their lifetime in the herd. However, whereas we consider a single generation of cows, Boichard et al. (2013) accrued benefits (and costs) for multiple generations over differing time periods. They showed that even after 10 yr, breakeven genotyping costs were still less than $€ 50$ when all females were inseminated with sexed semen and heifer calves were genomically tested. To fully assess the potential net benefit from using sexed semen, the other costs and benefits associated with the adoption of sexed semen must be considered.

Heifer sale prices after accounting for rearing costs (or MRH), had a large effect on net benefit. Similarly, both McCullock et al. (2013) and De Vries (2015) found heifer sale price to be the most influential factor in the cost-benefit of using sexed semen. The 3 scenarios tested by McCullock et al. (2013) included using sexed semen in heifers alone, in heifers and superior cows, and using sexed semen in heifers and superior cows, with the remainder mated to beef semen. Although McCullock et al. (2013) used a variable called "genetic gain from sexed semen" in their modeling, it was not linked to the herd's replacement rate, as in our study. Fetrow et al. (2007) found that when the price received for surplus heifers decreased by $22 \%$, using sexed semen was never profitable. However, that study only considered the value of extra heifers, not genetic gain. Our model found that sexed semen is likely to remain profitable even when MRH drops dramatically, because it considers the value of both genetic gain and surplus heifers. If MRH decreased to $\mathrm{A} \$ 200$ or lower, strategies for using sexed semen should be re-evaluated. For instance, there could be an opportunity to use genomic information to prioritize which animals should be inseminated with sexed semen (Boichard et al., 2013) and an opportunity to use beef semen in lower-genetic-merit individuals, assuming the price for a cross-bred calf is higher than that of a dairy calf (McCullock et al., 2013; Hjortø et al., 2015).

Although herds with high reproductive performance saw the highest net benefit from using sexed semen and genomic testing together, herds with moderate to high replacement rates and low reproductive performance saw a greater percentage increase in net benefit. This is likely to be because when reproductive performance is low, having only a limited number of heifers surplus to farm requirements limits the ability to apply selection pressure. Additionally, more non-AI-sired heifers of lower genetic merit are likely to be retained as replacements when selection pressure is low. Other than being able to evaluate whether the net benefit is positive for selecting heifer replacements, other nonfinancial reasons might influence a farmer's decision to use sexed semen. From a farm management point of view, heifer calves are associated with fewer incidences of dystocia than male calves. Maintaining a closed herd while ensuring there are enough replacements or while increasing herd size, or the desire to get replacements from heifers and cows with a perceived higher value may all influence a farmer's decision to use sexed semen (Fetrow et al., 2007). The opportunity to reduce the number of male calves is also an opportunity to alleviate growing animal welfare concerns from the public about the fate of male calves.

\section{Model Justifications}

Several of the assumptions made in this study differ from those of other studies. In our study, all 
heifer calves were assumed to be genomic tested. An alternative (for herds with prior access to $E B V_{P A}$ ) is a 2-stage selection strategy, with $\mathrm{EBV}_{\mathrm{PA}}$ used to identify candidates for genomic testing. This was not done in our study because a prior study (Calus et al., 2015) found that it was usually optimal to genomic test all heifers, unless herds had a very high number of selection candidates and a low replacement rate. It was also shown by Weigel et al. (2012) that, if the top 40 or $60 \%$ of animals were being selected, the best strategy was to genotype all selection candidates. Similarly, the decision to only use sexed semen for the first service differs from other studies (Hutchinson et al., 2013a,b), where it was recommended that sexed semen be used for the first 2 inseminations. Although we initially considered this, preliminary results showed that having 2 inseminations with sexed semen was never profitable in cows. In addition, consultation with service providers, breeding companies, and farmers showed that current practice in Australia is to use sexed semen only at the first service. As well as costs, another reason given was the challenge of monitoring heifers for subsequent estrus in our largely pasture-based systems. This study also did not consider that in a herd that has a history of making genetic progress, a heifer calf out of a heifer is genetically better than a heifer calf out of a cow.

A novel aspect of this paper is considering 2 populations of differing genetic merit (AI and non-AI calves) from which genotyped herd replacements are chosen. When calculating selection response, we assumed that the true genetic SD of the 2 populations are the same, $\sigma_{B P I}=\mathrm{A} \$ 90$. However, we assumed that the SD of $\mathrm{EBV}$ for BPI (the information that is used to calculate selection intensity) was different in the 2 populations, $\mathrm{A} \$ 45$ and $\mathrm{A} \$ 90$ for $\mathrm{AI}$ and non-AI calves, respectively. If this were not the case and the true genetic SD on the non-AI calf population were lower, we would expect the net benefit to be slightly reduced. Greater reductions would be seen where the net benefit is already marginal - typically scenarios with higher replacement rates as more non-AI calves are retained as replacements.

\section{Implications}

Being able to demonstrate that investing in genomic testing can quickly pay itself may help to increase the uptake of commercial genomic testing of heifers, which is especially useful for countries, such as Australia, where commercial uptake has been comparatively low compared with North America. The overall benefits of genomic testing are greater than the net benefit calculated in this study, as other opportunities exist to use genomic information on farm; such as to confirm parentage and in mating plans (i.e., Boichard et al., 2013; Hjortø et al., 2015). Having access to genomic information also gives greater flexibility in deciding when surplus heifers are sold, as replacements can be chosen with confidence at younger ages. The ability to demonstrate that investing in genomic testing generates a positive return from one aspect of its uses and, within a short time frame, may assist in the development of extension messages. For this reason, we considered only the value of having genomic information to make selection decisions and focused on showing that is possible to recoup the initial investment within the lifetime of the genotyped heifer cohort. This means we only calculated the genetic gain from heifer selection decisions in that generation. Obviously, the benefits of more accurately selecting replacement heifers are cumulative and permanent and continue to increase over time. Whether a farmer will see a positive return from using genomic information to guide heifer selection decisions is largely influenced by the intensity with which selection pressure can be applied to candidates. At the farm level, many factors, such as the overall herd reproduction rate, mortality rates, and heifer and cow conception rates, influence the number of heifer selection candidates available and number of replacements required. Replacement rate may also be affected by decisions made about voluntary culling of cows. Many parameters are sensitive to small changes and interactions occur between parameters. This means that there is no one-size-fits-all answer as to whether investing in genomic testing of heifers to guide replacement decisions with or without sexed semen usage is profitable at current prices. This is supported by others such as Fetrow et al. (2007), who also found that the optimal use of sexed semen is influenced by many economic and biological factors. Until reliabilities of $\mathrm{EBV}_{\mathrm{G}}$ are higher and costs of sexed semen and genomic testing are further reduced, the decision to genomic test should be made on an individual farm basis.

\section{CONCLUSIONS}

Genomic testing of heifer selection candidates may be a profitable investment. Under current Australian parameters, the net benefit of genomic testing is expected to range from $\mathrm{A} \$ 204$ to $\mathrm{A} \$ 1,124$ for a herd with median reproductive performance. Herd reproduction and replacement rates and the availability of $\mathrm{EBV}_{\mathrm{PA}}$ have the most influence on whether the investment in genomic testing is profitable at current prices. Concurrent adoption of sexed semen and genomic testing offers an opportunity to increase net benefit more than genomic testing only, but is highly influenced by the 
individual parameters of a farm. Currently, benefits from using sexed semen, in conjunction with genomic testing, are most likely to be seen when usage is restricted to heifers. Wide-scale use of sexed semen, in conjunction with genomic testing, will largely depend on achieving conception rates that are comparable to those of conventional semen and the sale price of these surplus heifers. Over time, as the cost of genomic testing decreases and genomic reliabilities increase, the proportion of dairy farmers for whom genomic testing is profitable will increase. The fact that a positive net benefit is seen even if genomic information is only used once in an animal's lifetime will facilitate the development of strong messages to increase uptake of this technology.

\section{ACKNOWLEDGMENTS}

This work was completed as part of the ImProving Herds Project. ImProving Herds is funded by the Gardiner Foundation (Melbourne, Australia) and Dairy Australia (Melbourne, Australia) with collaborative support from Agriculture Victoria (Victoria, Australia), DataGene Ltd. (Melbourne, Australia), Holstein Australia (Melbourne, Australia), and the National Herd Improvement Association of Australia (Werribee, Australia). The authors gratefully acknowledge the contributions of Peter Williams (DataGene Ltd., Melbourne, Australia), Tim Luke (Agriculture Victoria, Victoria, Australia), Richard Shepherd (Herd Health Pty Ltd, Maffra, Australia), and Tom Newton (MaxCare, Port Fairy, Australia) in estimating heifer rearing costs and sale prices and the insightful discussions with Mekonnen Haile-Mariam (Agriculture Victoria, Victoria, Australia) on reproductive parameters. We also extend our appreciation to the anonymous reviewers for their valuable contribution.

\section{REFERENCES}

Berry, D. P., E. Wall, and J. E. Pryce. 2014. Genetics and genomics of reproductive performance in dairy and beef cattle. Animal 8:105-121. https://doi.org/10.1017/S1751731114000743.

Bijma, P. 2012. Accuracies of estimated breeding values from ordinary genetic evaluations do not reflect the correlation between true and estimated breeding values in selected populations. J. Anim. Breed. Genet. 129:345-358. https://doi.org/10.1111/j.1439-0388 .2012.00991.x.

Boichard, D., R. Dassonneville, S. Mattalia, V. Ducrocq, and S. Fritz. 2013. All cows are worth to be genotyped. Interbull Bull. 47:256260.

Butler, S. T., I. A. Hutchinson, A. R. Cromie, and L. Shalloo. 2014. Applications and cost benefits of sexed semen in pasture-based dairy production systems. Animal 8:165-172. https://doi.org/10 .1017/S1751731114000664

Byrne, T. J., B. F. S. Santos, P. R. Amer, D. Martin-Collado, J. E. Pryce, and M. Axford. 2016. New breeding objectives and selection indexes for the Australian dairy industry. J. Dairy Sci. 99:81468167. https://doi.org/10.3168/jds.2015-10747.

Cabrera, V. E. 2009. When to use sexed semen in heifers. Paged 1-9 in Proc. Dairy Cattle Reprod. Counc. Annu. Conv., Minneapolis, MN, and Boise, ID; DCRC, New Prague, MN.

Calus, M. P., P. Bijma, and R. Veerkamp. 2015. Evaluation of genomic selection for replacement strategies using selection index theory. J. Dairy Sci. 98:6499-6509. https://doi.org/10.3168/jds.2014-9192.

Dairy Australia. 2013. Heifers on Target: A guide to growing more productive heifers. Dairy Australia, Melbourne.

De Vries, A. 2015. The economics of sexed semen in dairy heifers and cows. Page 1-11. Institute of Food and Agricultural Sciences Extension, University of Florida, Gainesville. Accessed Dec. 1, 2017 http://edis.ifas.ufl.edu/an214.

DeJarnette, J. M., R. L. Nebel, and C. E. Marshall. 2009. Evaluating the success of sex-sorted semen in US dairy herds from on farm records. Theriogenology 71:49-58. https://doi.org/10.1016/j .theriogenology.2008.09.042.

Falconer, D. S. 1989. Introduction to Quantitative Genetics. 3rd ed. Longman Scientific and Technical, New York, NY.

Fetrow, J., M. Overton, and S. Eicker. 2007. Sexed Semen: Where do we stand and where are we going. Pages 39-60 in Proc. 2007 Western Dairy Management Conf., Reno, NV. Oregon State University, Corvallis.

Granleese, T., S. A. Clark, A. A. Swan, and J. H. J. Werf. 2015. Increased genetic gains in sheep, beef and dairy breeding programs from using female reproductive technologies combined with optimal contribution selection and genomic breeding values. Genet. Sel. Evol. 47:70. https://doi.org/10.1186/s12711-015-0151-3.

Healy, A. A., J. K. House, and P. C. Thomson. 2013. Artificial insemination field data on the use of sexed and conventional semen in nulliparous Holstein heifers. J. Dairy Sci. 96:1905-1914. https:// doi.org/10.3168/jds.2012-5465.

Hjortø, L., J. F. Ettema, M. Kargo, and A. C. Sørensen. 2015. Genomic testing interacts with reproductive surplus in reducing genetic lag and increasing economic net return. J. Dairy Sci. 98:646-658. https://doi.org/10.3168/jds.2014-8401.

Hopkins, I. R., and J. W. James. 1978. Theory of nucleus breeding schemes with overlapping generations. Theor. Appl. Genet. 53:1724.

Hutchinson, I. A., L. Shalloo, and S. T. Butler. 2013a. Expanding the dairy herd in pasture-based systems: The role of sexed semen use in virgin heifers and lactating cows. J. Dairy Sci. 96:6742-6752. https://doi.org/10.3168/jds.2012-6476.

Hutchinson, I. A., L. Shalloo, and S. T. Butler. 2013b. Expanding the dairy herd in pasture-based systems: The role for sexed semen use on virgin heifers. J. Dairy Sci. 96:1312-1322. https://doi.org/10 $.3168 /$ jds.2012-6126

Izzo, M. 2015. Sex-sorted semen: the potential reproductive game changer. Pages 145-154 in Proc. Combined Aust. Cattle Vet. Aust. Sheep Vet. Annu. Conf., Hobart, TAS, Australia. Aust. Cattle Vet., QLD, Australia.

McCullock, K., D. L. K. Hoag, J. Parsons, M. Lacy, G. E. Seidel Jr. and W. Wailes. 2013. Factors affecting economics of using sexed semen in dairy cattle. J. Dairy Sci. 96:6366-6377. https://doi.org/ $10.3168 /$ jds.2013-6672.

Mc Hugh, N., T. H. E. Meuwissen, A. R. Cromie, and A. K. Sonesson. 2011. Use of female information in dairy cattle genomic breeding programs. J. Dairy Sci. 94:4109-4118. https://doi.org/10.3168/jds $.2010-4016$.

Morton, J. 2011. InCalf Fertility Data Project 2011. Dairy Australia and Harris Park Group, Melbourne, VIV, Australia.

Noonan, E. J., J. C. Kelly, and D. S. Beggs. 2016. Factors associated with fertility of nulliparous dairy heifers following a 10 day fixedtime artificial insemination program with sex-sorted and conventional semen. Aust. Vet. J. 94:145-148. https://doi.org/10.1111/ avj. 12430

Partnerships Victoria. 2003. Technical Note - Use of Discount Rates in Partnerships Victoria Projects. Department of Treasury and Finance, Melbourne. Accessed Jun. 30, 2017. www.partnerships .vic.gov.au. 
Pryce, J. E., P. Douglas, C. M. Reich, A. J. Chamberlain, P. J. Bowman, T. T. T. Nguyen, B. A. Mason, C. P. Prowse-Wilkins, G. J. Nieuwhof, T. Hancock, M. Shaffer, and B. J. Hayes. 2017. Reliabilities of Australian dairy genomic breeding values increase through the addition of genotyped females with excellent phenotypes. Pages 133-136 in Proc. Assoc. Advmt. Anim. Breed. Genet. 22. AAABG, Townsville, Australia.

Pryce, J. E., M. Goddard, H. Raadsma, and B. Hayes. 2010. Deterministic models of breeding scheme designs that incorporate genomic selection. J. Dairy Sci. 93:5455-5466. https://doi.org/10.3168/jds 2010-3256

Pryce, J. E., and B. J. Hayes. 2012. A review of how dairy farmers can use and profit from genomic technologies. Anim. Prod. Sci. 52:180-184. https://doi.org/10.1071/AN11172.

Pryce, J. E., B. J. Hayes, and M. Goddard. 2012. Genotyping dairy females can improve the reliability of genomic selection for young bulls and heifers and provide farmers with new management tools. Proceedings of ICAR 38th Biennial Meeting, Cork, Ireland. International Committee for Animal Recording (ICAR), Rome, Italy.

R Development Core Team. 2017. R: A language and environment for statistical computing. R Foundation for Statistical Computing, Vienna, Austria. http://www.R-project.org/.

Visscher, P. M., J. A. Woolliams, D. Smith, and J. L. Williams. 2002. Estimation of pedigree errors in the UK dairy population using microsatellite markers and the impact on selection. J. Dairy Sci. 85:2368-2375.

Weigel. K. A., P. C. Hoffman, W. Herring, and T. J. Lawlor Jr. 2012. Potential gains in lifetime net merit from genomic testing of cows, heifers, and calves on commercial dairy farms. J. Dairy Sci. 95:2215-2225. https://doi.org/10.3168/jds.2011-4877. 